\title{
Ne pas sous-estimer le sérieux de la réflexion des couples
}

\section{Jean Martin}

Ancien membre de la Commission nationale d'éthique, membre de la rédaction

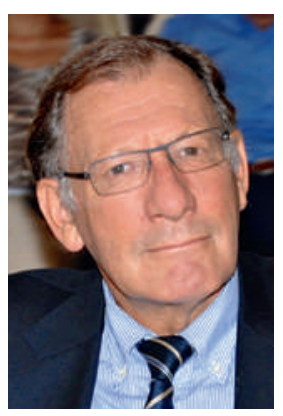

Le 14 juin prochain, le peuple se prononce sur une modification de l'article 119 de la Constitution fédérale. Cette modification est rendue nécessaire dans l'optique d'une disposition nouvelle de la loi sur la procréation médicalement assistée (PMA) autorisant le diagnostic préimplantatoire (DPI). On sait que le DPI est admis dans des pays qui nous sont proches - où aujourd'hui les couples suisses sont contraints de se rendre pour bénéficier de cette technique.

Certains jugent qu'il y a là une dérive inacceptable de type eugénique et critiquent une poussée vers l'enfant parfait (alors qu'on parle d'éviter des maladies graves). Il faut rappeler que le diagnostic prénatal (DPN ) est pratiqué chez nous: examens durant la grossesse, avec la possibilité d'une interruption (IG) si une anomalie est détectée; or, l'éventualité de l'IG dépend de la seule détermination de la femme jusqu'à la $12^{\mathrm{e}}$ semaine, le peuple suisse l'a voulu ainsi en 2002.

Les opposants semblent penser que le choix du DPN est moins grave que celui du DPI où, in vitro, avant donc toute grossesse, on choisit un embryon qui ne porte pas le défaut génétique qui mènerait à une affection sévère. Mais quelle considération accordent-ils à la réalité de la vie de nos contemporains? Aujourd'hui déjà, des décisions sont prises que personne ne conteste: indépendamment de toute technique médicale, les couples choisissent le moment auquel ils souhaitent des enfants et leur nombre. Avoir cinq enfants représente aujourd'hui une grande famille alors que biologiquement la femme peut en avoir une vingtaine; de très nombreux enfants ne naissent pas alors qu'ils pourraient naître.

Ainsi, alors que des options qui sont des manières de "décider qui vit et qui meurt» interviennent quotidiennement au sein des familles, pour des raisons très diverses, est-il défendable de refuser que la médecine assiste celles qui sont à grand risque d'avoir des enfants porteurs d'importants déficits? Au nom de quoi? De l'idée qu'on doit les laisser dépendre d'une «nature» qui joue aux dés avec leurs gamètes?

Autre argument, la crainte que les personnes porteuses de handicaps, si elles deviennent plus rares, ne soient plus acceptées de la même manière par la société. Cette préoccupation mérite tout notre respect. Cela étant, tendrait-on à dire que, pour être solidaire, une collecti- vité devrait comporter un tant pour cent de personnes handicapées? Inimaginable de préserver notre vivreensemble en interdisant d'éviter la naissance d'enfants porteurs de déficits. Dans un débat radiophonique récent, les représentants des familles concernées faisaient bien la part des choses; tout en donnant beaucoup d'amour à leur enfant handicapé, ils reconnaissaient souhaiter avoir par le DPI la possibilité d'un autre enfant qui n'aurait pas ce grave souci de vie.

On a entendu un philosophe dire qu'on ne saurait laisser à «l'arbitraire des parents» des choix tels que ceux qu'implique le DPI. Alors que, cela vient d'être rappelé, des choix de cet ordre sont constamment faits en toute liberté. J'ai été médecin officiel et crois aux rôles de l'Etat. Mais je crois aussi que l'Etat est bien avisé de faire preuve de retenue avant de vouloir régler la vie intime des citoyens. Une "élite» craindrait-elle que des personnes du commun prennent des déterminations frivoles, pour des motifs de pure convenance ? Propos du Dr Véronique Fournier, du Centre d'éthique de l'Hôpital Cochin, à Paris, sur ses entretiens avec des couples: «La conception même d'un garde-fou mis en place par la société pour protéger les enfants [à naître] des excès de leurs parents leur était incompréhensible. Qui mieux qu'eux connaissait le vrai prix à payer de tel ou tel choix? En revanche, ils comprenaient que leur médecin ait besoin de réfléchir avec d'autres afin de les guider au mieux.» Dans un pays comme le nôtre qui valorise tellement la liberté de chacun, veut-on dire que telle commission officielle ou tels fonctionnaires seront plus «intelligents» que les couples dans ces décisions toujours lourdes?

La médecine et ses avancées lancent de sérieux défis, c'est clair. Cela exige d'y réfléchir au plan éthique, Mais il est nécessaire que ces experts réfléchissent aussi en termes de réalité et de bon sens quotidien. Au risque d'être indûment terre-à-terre, un élément encore: en refusant le DPI, avec l'idée d'être meilleur que les voisins, on maintient une situation où les personnes aisées obtiennent sans autre cette prestation à l'étranger, mais où cela n'est pas envisageable pour les gens modestes. Argument matériel, mais peut-on le balayer du dos de la main? Là aussi, il s'agit de maintenir une société un peu solidaire. 